\title{
Necessary and possible interaction in a 2-maxitive Sugeno integral model
}

\begin{abstract}
This paper proposes and studies the notion of interaction between two criteria in a 2-maxitive Sugeno integral model. Within the framework of binary alternatives, we give a necessary and sufficient condition for preferential information on binary alternatives to be representable by a 2-maxitive Sugeno integral model. Using this condition, we show that it is always possible to choose a numerical representation, for which all the interaction indices are strictly positive. Outside the framework of binary alternatives, by introducing of binary variables, we propose a MILP allowing to test whether an ordinal preference information is representable by a 2-maxitive Sugeno integral model and whether the interpretation of the interaction indices is ambiguous or not. We illustrate our results with examples.
\end{abstract}

Keywords: Binary alternatives, Sugeno integral model, Interaction indices, 2-maxitive capacity

\section{Introduction}

The Sugeno integral was introduced in [18] and it is an aggregation function in the ordinal approach to decision making. There are numerous applications of the Sugeno integral in the decision making [7,9], and the problem of identification of fuzzy measures, based on which fuzzy integrals are defined, has attracted substantial attention $[1,2,10]$.

In the context of a 2-additive Choquet integral model, the interaction index between two criteria coincides with the Möbius mass of this pair [15]. By analogy, in the context of a 2-maxitive Sugeno integral, in this paper we propose a definition of the interaction index between two criteria.

In [15], we find necessary and sufficient conditions for a preferential information on a set of binary alternatives to be represented by a 2-additive Choquet integral model. We give a similar result with the 2-maxitive Sugeno integral model.

In [14], it is proven that in the framework of binary alternatives, if the preferential information contains no indifference, and if it is representable by a 2additive Choquet integral model, then it is always possible to represent these preferences by a strict positive interaction. We obtain a similar result with the 2-maxitive Sugeno integral.

Outside the framework of binary alternatives, in [15] we find a simple LP allowing us to test whether ordinal preference information is representable by a 2-additive Choquet integral model and whether the interpretation of interaction 
indices is or not ambiguous. Outside the framework of binary alternatives, using the linearization of the min and max functions, we propose a MILP allowing to test whether an ordinal preference information is representable by a 2-maxitive Sugeno integral model and whether the interpretation of the interaction indices is or not ambivalent.

This paper is organized as follows. After having recalled in Section 2 some basic elements on the model of the Sugeno integral in MCDM, in Section 3, we use a classic example to argue that the usual interpretation of interaction indices is not always convincing. In Section 4, we give our main results. Outside the framework of binary alternatives, by introduicing binary variables, we propose a MILP model allowing to test whether an ordinal preference information is representable by a 2-maxitive Sugeno integral model and whether the interaction is necessary or not. We illustrate our results with an example in Section 6, and we end by a conclusion.

\section{Notations and definitions}

\subsection{The framework}

Let $N=\{1,2, \ldots, n\}$ be a set of $n$ criteria and $L_{i}$ the evaluation scale for the criterion $i \in N$. We denote by $0_{i}$ (resp. $1_{i}$ ) the smallest (resp. biggest) element of $L_{i}$. An alternative is a vector $x=\left(x_{1}, \cdots, x_{n}\right) \in L_{1} \times L_{2} \times \cdots \times L_{n}$ where $x_{i}$ is the ordinal evaluation of the alternative with respect to the criterion $i \in N$. The criteria are recoded numerically using, for all $i \in N$, a function $u_{i}$ from $L_{i}$ into $\mathbb{R}$.

\section{$2.2 \quad$ Sugeno integral}

The Sugeno integral $[5,7,18]$ is an aggregation function known in MCDM. It is based on the notion of capacity $[4,16]$ defined as a function $\mu$ from the powerset $2^{N}$ into $L$ such that:

- $\mu(\emptyset)=0$,

- $\mu(N)=1$,

- $\forall S, T \in 2^{N},[S \subseteq T \Longrightarrow \mu(S) \leq \mu(T)]$ (monotonicity).

For an alternative $x=\left(x_{1}, \cdots, x_{n}\right) \in L_{1} \times L_{2} \times \cdots \times L_{n}$, the expression of the Sugeno integral w.r.t. a capacity $\mu$ is given by:

$$
S_{\mu}(u(x))=\bigvee_{i=1}^{n}\left(u_{\sigma(i)}\left(x_{\sigma(i)}\right) \wedge \mu\left(N_{\sigma(i)}\right)\right)
$$

where $u(x)=\left(u_{1}\left(x_{1}\right), \cdots, u_{n}\left(x_{n}\right)\right), \sigma$ is a permutation on $N$ such that: $N_{\sigma(i)}=\{\sigma(i), \cdots, \sigma(n)\}$ and $u_{\sigma(1)}\left(x_{\sigma(1)}\right) \leq u_{\sigma(2)}\left(x_{\sigma(2)}\right) \leq \cdots \leq u_{\sigma(n)}\left(x_{\sigma(n)}\right)$. 
Remark 1. The Sugeno integral is equivalent to the following expression (see [13])

$$
S_{\mu}(u(x))=\bigvee_{A \subseteq N}\left(\left(\bigwedge_{i \in A} u_{i}\left(x_{i}\right)\right) \wedge \mu(A)\right) .
$$

The ordinal Möbius transform [11] $m^{\mu}$ of $\mu$ is defined by:

$$
m^{\mu}(S)= \begin{cases}\mu(S) & \text { if } \mu(S)>\bigvee_{T \subsetneq S} \mu(T) \\ 0 \quad & \text { otherwise }\end{cases}
$$

The definition of a capacity generally requires $2^{n}-2$ coefficients which are the values of $\mu$ for all subsets of $N$. When $n$ is large, this determination becomes difficult. This is why the concept of $k$-maxitive capacity, where $k$ is an integer between 1 and $n$, has been introduced in order to reduce the number of parameters of $\mu$ to be determined.

Definition 1. A capacity $\mu$ is said to be $k$-maxitive [17, 3] if we have

$$
\mu(S)=\bigvee_{\substack{T \subseteq S \\|T| \leq k}} \mu(T) \text { for all } S \subseteq N .
$$

Remark 2. - $k$-maxitive capacities are thus completely determined by their values on the sets with at most $k$ elements.

- A Sugeno integral defined with respect to a $k$-maxitive capacity is also said to be $k$-maxitive.

- It is not difficult to see from (4) that for a $k$-maxitive Sugeno integral $S_{\mu}$, $m^{\mu}(S)=0$ for all $S \subseteq N$ such that $|S|>k$, and thus $S_{\mu}$ can be expressed as a supremum of terms with at most $k$ variables.

The following proposition gives a simplified expression of the $k$-maxitive Sugeno integral

Proposition 1. If a capacity $\mu$ is $k$-maxitive, then we have:

$$
S_{\mu}(u(x))=\bigvee_{\substack{A \subseteq N \\|A| \leq k}}\left(\left(\bigwedge_{i \in A} u_{i}\left(x_{i}\right)\right) \wedge \mu(A)\right)
$$

Proof. Let us assume that a capacity $\mu$ is $k$-maxitive. We then have $\mu(A)=\bigvee_{\substack{B \subseteq A \\ \mid B \backslash \leq k}} \mu(B)$ for $|A| \geq k+1$.

Since $\bigwedge_{i \in A} u_{i}\left(x_{i}\right) \geq \bigwedge_{i \in B} u_{i}\left(x_{i}\right)$ when $A \subseteq B$, so

$$
\bigvee_{\substack{A \subseteq N \\|A| \leq k}}\left(\left(\bigwedge_{i \in A} u_{i}\left(x_{i}\right)\right) \wedge \mu(A)\right) \geq \bigvee_{\substack{A \subseteq N \\|A| \geq k+1}}\left(\left(\bigwedge_{i \in A} u_{i}\left(x_{i}\right)\right) \wedge \mu(A)\right)
$$


Thus we have:

$$
\begin{aligned}
S_{\mu}(x) & =\bigvee_{A \subseteq N}\left(\left(\bigwedge_{i \in A} u_{i}\left(x_{i}\right)\right) \wedge \mu(A)\right) \\
& =\left[\bigvee_{\substack{A \subseteq \\
|A| \leq k}}\left(\left(\bigwedge_{i \in A} u_{i}\left(x_{i}\right)\right) \wedge \mu(A)\right)\right] \bigvee\left[\bigvee_{\substack{A \subseteq N \\
|A| \geq k+1}}\left(\left(\bigwedge_{i \in A} u_{i}\left(x_{i}\right)\right) \wedge \mu(A)\right)\right] \\
& =\bigvee_{\substack{A \subseteq N \\
|A| \leq k}}\left(\left(\bigwedge_{i \in A} u_{i}\left(x_{i}\right)\right) \wedge \mu(A)\right)
\end{aligned}
$$

Remark 3. For $k=1$, the Sugeno integral simplifies in the form of a prioritized maximum [6]:

$$
S_{\mu}(u(x))=\bigvee_{i \in N}\left(u_{i}\left(x_{i}\right) \wedge \mu_{i}\right)
$$

Remark 4. For $k=2$, the 2-maxitive Sugeno integral is given by:

$$
S_{\mu}(u(x))=\left[\bigvee_{i \in N}\left(u_{i}\left(x_{i}\right) \wedge \mu_{i}\right)\right] \bigvee\left[\bigvee_{i, j \in N}\left(u_{i}\left(x_{i}\right) \wedge u_{j}\left(x_{j}\right) \wedge \mu_{i j}\right)\right]
$$

In the context of a 2-additive Choquet integral, the interaction index between two criteria coincides with their Möbius transform [15]. By analogy, in the framework of a 2-maxitive Sugeno integral, we propose below a definition of the interaction index between two criteria. Indeed, we propose that $I_{i j}^{\mu}=m_{i j}^{\mu}$ for all $i, j \in N$.

Definition 2. The interaction index w.r.t. a 2-maxitive capacity $\mu$ is defined by:

$$
I_{i j}^{\mu}= \begin{cases}\mu_{i j} & \text { if } \mu_{i j}>\mu_{i} \vee \mu_{j} \\ 0 & \text { otherwise }\end{cases}
$$

Remark 5. Given a capacity $\mu$, it is usual to interpret the interaction as follows:

- If $I_{i j}^{\mu}>0$, then we say that criterion $i$ and $j$ are complementary( or in positive synergy) w.r.t $\mu$.

- If $I_{i j}^{\mu}=0$, then we say that criterion $i$ and $j$ are independent w.r.t $\mu$.

Our definition for the 2-maxitive case implies that negative interactions are not taken into account.

\section{A motivating example}

We consider the following example inspired by [?]. Four students are evaluated on three subjects Mathematics (M), Statistics (S) and Language skills (L). All marks are taken from the same scale, from 0 to 1 . The evaluations of these students are given by the Table 1 . 


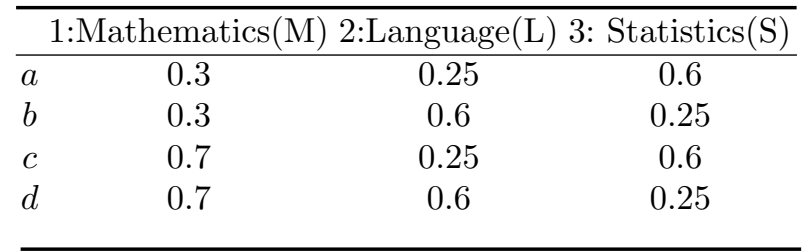

Table 1: Evaluations students

To select the best students, the Dean of the faculty expresses his/her preferences where the notation $x P y$ means $x$ is strictly preferred to $y$. For a student bad in Mathematics, Language is more important that Statistics, so that

$$
a P b
$$

for a student good in Mathematics, Statistics is more important that Language, so that

$$
d P c \text {. }
$$

It is not possible to model the two preferences $a P b$ and $d P c$ by an 1-maxitive Sugeno integral model. Indeed we have:

$$
\begin{aligned}
& S_{\mu}(a)=\left(u_{M}(0.3) \wedge \mu_{1}\right) \vee\left(u_{L}(0.25) \wedge \mu_{2}\right) \vee\left(u_{S}(0.6) \wedge \mu_{3}\right) \\
& S_{\mu}(b)=\left(u_{M}(0.3) \wedge \mu_{1}\right) \vee\left(u_{L}(0.6) \wedge \mu_{2}\right) \vee\left(u_{S}(0.25) \wedge \mu_{3}\right) \\
& S_{\mu}(c)=\left(u_{M}(0.7) \wedge \mu_{1}\right) \vee\left(u_{L}(0.25) \wedge \mu_{2}\right) \vee\left(u_{S}(0.6) \wedge \mu_{3}\right) \\
& S_{\mu}(d)=\left(u_{M}(0.7) \wedge \mu_{1}\right) \vee\left(u_{L}(0.6) \wedge \mu_{2}\right) \vee\left(u_{S}(0.25) \wedge \mu_{3}\right)
\end{aligned}
$$

We then have: $\left(u_{M}(0.7) \wedge \mu_{1}\right) \vee S_{\mu}(a)=\left(u_{M}(0.3) \wedge \mu_{1}\right) \vee S_{\mu}(c)$ and

$$
\left(u_{M}(0.7) \wedge \mu_{1}\right) \vee S_{\mu}(b)=\left(u_{M}(0.3) \wedge \mu_{1}\right) \vee S_{\mu}(d) .
$$

Therefore we have: $a P b \Longrightarrow S_{\mu}(a)>S_{\mu}(b)$,

$$
\begin{aligned}
& \Longrightarrow\left(u_{M}(0.7) \wedge \mu_{1}\right) \vee S_{\mu}(a) \geq\left(u_{M}(0.7) \wedge \mu_{1}\right) \vee S_{\mu}(b), \\
& \Longrightarrow\left(u_{M}(0.3) \wedge \mu_{1}\right) \vee S_{\mu}(c) \geq\left(u_{M}(0.3) \wedge \mu_{1}\right) \vee S_{\mu}(d), \\
& \Longrightarrow S_{\mu}(c) \geq S_{\mu}(d), \\
& \Longrightarrow \operatorname{not}(d P c) \text {. Contradiction with preference } d P c .
\end{aligned}
$$

Let us assume that the scale of evaluation $[0,1]$ corresponds to the utility function associated to each subject, i.e., $u_{M}(0.3)=0.3, u_{M}(0.7)=0.7, u_{L}(0.25)=$ $0.25, u_{L}(0.6)=0.6, u_{S}(0.25)=0.25$ and $u_{S}(0.6)=0.6$. In this case, the preferences $a P b$ and $d P c$, are now representable by a 2-maxitive integral w.r.t. any capacity given in Table 2 below. We chose eight capacities compatible with these preferences (Cap. for short in Table 2) in order to illustrate the fact that the sign of an interaction index is strongly dependent upon to the chosen capacity. 
In this illustration, the interpretation of the interaction between two criteria is not easy. For instance, w.r.t. the 2-maxitive capacity, the interaction between Mathematics and Statistics, $I_{M S}^{\mu}$, could be strictly positive (Cap. 2, Cap. 7, Cap. 8) or null (Cap. 1, Cap. 3, Cap. 4, Cap. 5, Cap. 6). Thus, from the preferences given by the DM, it is not obvious whether the subjects Mathematics and Statistics are complementary or independent. This conclusion is still valid concerning the interaction $I_{L S}^{\mu}$ between Language and Statistics. Indeed, this interaction is strictly positive (see Cap. 2, Cap. 3, Cap. 4, Cap. 6 and Cap. 7), and null (see Cap. 1 and Cap. 5).

\begin{tabular}{|c|c|c|c|c|c|c|c|c|}
\hline & \multicolumn{8}{|c|}{ Cap. 1 Cap. 2 Cap. 3 Cap. 4 Cap. 5 Cap. 6 Cap. 7 Cap. 8} \\
\hline$\mu_{M}$ & 0 & 0 & 0.3 & 0.5 & 0.3 & 0 & 0 & 0 \\
\hline$\mu_{L}$ & 0 & 0 & 0 & 0 & 0 & 0 & 0 & 0 \\
\hline$\mu_{S}$ & 0.5 & 0.4 & 0.5 & 0.5 & 0.5 & 0.5 & 0.49 & 0.49 \\
\hline$\mu_{M L}$ & 1 & 1 & 0.6 & 0.6 & 1 & 0.6 & 0.6 & 1 \\
\hline$\mu_{M S}$ & 0.5 & 0.5 & 0.5 & 0.5 & 0.5 & 0.5 & 0.5 & 0.5 \\
\hline$\mu_{L S}$ & 0.5 & 0.5 & 1 & 1 & 0.5 & 1 & 1 & 0.49 \\
\hline$S_{\mu}(a)$ & 0.5 & 0.4 & 0.5 & 0.5 & 0.5 & 0.5 & 0.49 & 0.49 \\
\hline$S_{\mu}(b)$ & 0.3 & 0.3 & 0.3 & 0.3 & 0.3 & 0.3 & 0.3 & 0.3 \\
\hline$S_{\mu}(c)$ & 0.5 & 0.5 & 0.5 & 0.5 & 0.5 & 0.5 & 0.5 & 0.5 \\
\hline$S_{\mu}(d)$ & 0.6 & 0.6 & 0.6 & 0.6 & 0.6 & 0.6 & 0.6 & 0.6 \\
\hline$I_{M L}^{\mu}$ & 1 & 1 & 0.6 & 0.6 & 1 & 0.6 & 0.6 & 1 \\
\hline$I_{M S}^{\mu}$ & 0 & 0.5 & 0 & 0 & 0 & 0 & 0.5 & 0.5 \\
\hline$I_{L S}^{\mu}$ & 0 & 0.5 & 1 & 1 & 0 & 1 & 1 & 0 \\
\hline
\end{tabular}

Table 2: A set of eight 2-maxitive capacities compatible with the preferences $a P b$ and $d P c$.

Depending on the numerical representation $\mu$, the interaction index can be null or strictly positive, this led to the definition of the notion of necessary and possible interaction, as introduced in $[12,14]$ for the Choquet integral model.

\section{Necessary and possible interaction}

In the sequel, we will suppose that the DM is able to compare a number of alternatives in terms of strict preference $(P)$ or indifference $(I)$. The idea is to ask to the DM its preferences by comparing some elements of $X$. We then obtain the binary relations $P$ and $I$ defined as follows.

Definition 3. An ordinal preference information $\{P, I\}$ on $X$ is given by:

$$
P=\{(x, y) \in X \times X: D M \text { strictly prefers } x \text { to } y\},
$$


$I=\{(x, y) \in X \times X: D M$ is indifferent between $x$ and $y\}$.

We say that $\{P, I\}$ is representable by a 2-maxitive Sugeno integral model, if there exists a 2-maxitive capacity $\mu$ such that: for all $x, y \in X$,

$$
\begin{aligned}
& (x, y) \in P \Longrightarrow S_{\mu}(u(x))>S_{\mu}(u(y)) \\
& (x, y) \in I \Longrightarrow S_{\mu}(u(x))=S_{\mu}(u(y)) .
\end{aligned}
$$

The set of all 2-maxitive capacities used to represent the preference information at hand will be denoted $S_{2-\max }(P, I)$. When there is no ambiguity on the underlying preference information, we will simply write $S_{2 \text {-max }}$.

The following definition of necessary and possible interactions will be central in the rest of this text.

Definition 4. Let be $i, j \in N$ be two distinct criteria, We say that:

1. there exists a possible complementary (resp. independence) between $i$ and $j$ if there exists a capacity $\mu \in S_{2-\max }$ such that $I_{i j}^{\mu}>0$ (resp. $I_{i j}^{\mu}=0$ );

2. there exists a necessary complementary (resp. independence) between $i$ and $j$ if $I_{i j}^{\mu}>0$ (resp. $I_{i j}^{\mu}=0$ ) for all capacity $\mu \in S_{2-\max }$.

Remark 6. Let $i, j \in N$ be two distinct criteria.

- If there exists a necessary complementary (resp. independence) between $i$ and $j$, then there exists a possible complementary (resp. independence) between $i$ and $j$.

- If there is no necessary complementary (resp. independence) between $i$ and $j$, then there exists a possible independence (resp. synergy) between $i$ and $j$.

\section{Necessary and possible interaction with binary alternatives}

\subsection{Framework}

We assume that the DM is able to identify on each criterion $i \in N$ two reference levels $1_{i}$ and $0_{i}$ :

- the level $0_{i}$ in $L_{i}$ is considered as a neutral level and we set $u_{i}\left(0_{i}\right)=0$;

- the level $1_{i}$ in $L_{i}$ is considered as a good level and we set $u_{i}\left(1_{i}\right)=1$.

For a subset $S \subseteq N$ we define the alternative $a_{S}=\left(1_{S} ; 0_{-S}\right)$ such that $a_{i}=1_{i}$ if $i \in S$ and $a_{i}=0_{i}$ otherwise. Our work is based on the set $\mathcal{B}$ that we can find in [15] and is defined as following.

Definition 5. The set of binary alternatives is defined by $\mathcal{B}=\left\{0_{N},\left(1_{i}, 0_{N-i}\right),\left(1_{i j}, 0_{N-i j}\right): i, j \in N, i \neq j\right\}$

where 
- $0_{N}=\left(1_{\emptyset}, 0_{N}\right)=: a_{0}$ is an alternative considered neutral on all criteria.

- $\left(1_{i}, 0_{N-i}\right)=: a_{i}$ is an alternative considered satisfactory on criterion $i$ and neutral on the other criteria.

- $\left(1_{i j}, 0_{N-i j}\right)=: a_{i j}$ is an alternative considered satisfactory on criteria $i$ and $j$ and neutral on the other criteria.

Remark 7. For any 2-maxitive capacity $\mu$, we have:

$$
S_{\mu}\left(u\left(a_{0}\right)\right)=0 ; S_{\mu}\left(u\left(a_{i}\right)\right)=\mu_{i} ; S_{\mu}\left(u\left(a_{i j}\right)\right)=\mu_{i j} .
$$

We add to this ordinal information a relation $M$ modeling the relation of monotonicity between binary alternatives, and allowing us to ensure the satisfaction of the monotonicity conditions $\mu_{i} \geq 0$ and $\mu_{i j} \geq \mu_{i}$ for a capacity $\mu$. For $(x, y) \in\left\{\left(a_{i}, a_{0}\right): i \in N\right\} \cup\left\{\left(a_{i j}, a_{i}\right): i, j \in N, i \neq j\right\}, x M y$ if not $(x(P \cup I) y)$.

\subsection{Results}

The following proposition gives a necessary and sufficient condition for an ordinal preference information on $\mathcal{B}$ containing no indifference to be representable by a 2-maxitive integral model.

Proposition 2. Let $\{P, I\}$ be an ordinal preference information on $\mathcal{B}$ such that $I=\emptyset$. Then $\{P, I\}$ is representable by a 2-maxitive Sugeno integral if and only if the binary relation $P \cup M$ contains no strict cycle.

Proof. Necessity. Suppose that the ordinal preference information $\{P, I\}$ on $\mathcal{B}$ is representable by a Sugeno integral. So there exists a capacity $\mu \in S_{2 \text {-max }}$ such that $\{P, I\}$ is representable by $S_{\mu}$.

If $P \cup M$ contains a strict cycle, then there exists $x_{0}, x_{1}, \ldots, x_{r}$ on $\mathcal{B}$ such that $x_{0}(P \cup M) x_{1}(P \cup M) \ldots(P \cup M) x_{r}(P \cup M) x_{0}$ and there exists two elements $x_{i}, x_{i+1} \in\left\{x_{0}, x_{1}, \ldots, x_{r}\right\}$ such that $x_{i} P x_{i+1}$. Since $\{P, I\}$ is representable by $S_{\mu}$, therefore $S_{\mu}\left(u\left(x_{0}\right)\right) \geq \ldots \geq S_{\mu}\left(u\left(x_{i}\right)\right)>S_{\mu}\left(u\left(x_{i+1}\right)\right) \geq \ldots \geq S_{\mu}\left(u\left(x_{0}\right)\right)$, then $S_{\mu}\left(u\left(x_{0}\right)\right)>S_{\mu}\left(u\left(x_{0}\right)\right)$, contradiction.

Sufficiency. Assume that $(P \cup M)$ contains no strict cycle. The proof of sufficiency consists to extend the relation $P$ to a total order on $\mathcal{B}$. This latter can be viewed as a partition of $\mathcal{B}$ elaborated by computing a topological sorting on $(P \cup M)$ detailed in Section 5.2. of [15].

Then there exists $\left\{\mathcal{B}_{0}, \mathcal{B}_{1}, \ldots, \mathcal{B}_{m}\right\}$ a partition of $\mathcal{B}$, builds by using a suitable topological sorting on $(P \cup M)[8]$.

We construct a partition $\left\{\mathcal{B}_{0}, \mathcal{B}_{1}, \ldots, \mathcal{B}_{m}\right\}$ as follows:

$\mathcal{B}_{0}=\{x \in \mathcal{B}: \forall y \in \mathcal{B}, \operatorname{not}[x(P \cup M) y]\}$,

$\mathcal{B}_{1}=\left\{x \in \mathcal{B} \backslash \mathcal{B}_{0}: \forall y \in \mathcal{B} \backslash \mathcal{B}_{0}, \operatorname{not}[x(P \cup M) y]\right\}$,

$\mathcal{B}_{i}=\left\{x \in \mathcal{B} \backslash\left(\mathcal{B}_{0} \cup \ldots \cup \mathcal{B}_{i-1}\right): \forall y \in \mathcal{B} \backslash\left(\mathcal{B}_{0} \cup \ldots \cup \mathcal{B}_{i-1}\right)\right.$, not $\left.[x(P \cup M) y]\right\}$, for all $i=1,2, \ldots, m$.

Let us define the mapping $\phi: \mathcal{B} \longrightarrow \mathcal{P}(N), f: \mathcal{P}(N) \longrightarrow \mathbb{R}, \mu: 2^{N} \longrightarrow[0,1]$ as 
follows: $\phi\left(a_{S}\right)=S$ for all $S \subseteq N, f(\phi(x))=\ell$ for all $\ell \in\{0,1, \ldots, m\}, \forall x \in \mathcal{B}_{\ell}$, $\mu_{\emptyset}=0, \mu_{i}=\frac{f_{i}}{\alpha}, \mu_{i j}=\frac{\bar{f}_{i j}}{\alpha}, \mu(S)=\bigvee_{i, j \in S} \mu_{i j}, \forall i, j \in N, \forall S \subseteq N$, where $f_{i}=f\left(\phi\left(a_{i}\right)\right), f_{i j}=f\left(\phi\left(a_{i j}\right)\right)$ and $\alpha=\bigvee_{i, j \in N}^{i, j \in S} \mu_{i j}$

The capacity $\mu$, defined like this is 2-maxitive by construction and the ordinal information $\{P, I\}$ is then representable by a 2 -maxitive Sugeno integral model $S_{\mu}$.

Given the ordinal preference information $\{P, I\}$ on $\mathcal{B}$, under the previous conditions, the following proposition shows that, it is always possible to choose in $S_{2-\max }(P, I)$, a capacity allowing all the interaction indices strictly positive. This result shows that positive synergy interaction is always possible for all pairs of criteria in a 2-maxitive Sugeno integral model if the ordinal information does not contain indifference. This condition is the same as that obtained in the case of the 2-additive Choquet integral [14].

Proposition 3. Let $\{P, I\}$ be an ordinal preference information on $\mathcal{B}$ such that $I=\emptyset$. Suppose that this information can be represented by the 2-maxitive Sugeno integral model. Then there exists a possible positive synergy between all pairs of criteria.

Proof. The partition $\left\{\mathcal{B}_{0}, \ldots, \mathcal{B}_{m}\right\}$ of $\mathcal{B}$ and the capacity $\mu$ are built as in the proof of Proposition 2: $\phi\left(a_{S}\right)=S$ for all $S \subseteq N$,

Let be $i, j \in N$, there exist $p, q, s \in\{1, \ldots, m\}$ such that $a_{i j} \in \mathcal{B}_{p}, a_{i} \in \mathcal{B}_{q}, a_{j} \in$ $\mathcal{B}_{s}$ with $p>q>0$ and $p>s>0$

The capacity $\mu$, defined like this is 2-maxitive by construction and in Proposition 2 we have proved that $S_{\mu}$ represent.

Moreover we have $f_{i j}=p, f_{i}=q, f_{j}=s$ with $p>q$ and $p>s$, therefore $p>q \vee s$, i.e., $\mu_{i j}>\mu_{i} \vee \mu_{j}$, then $I_{i j}^{\mu}=\mu_{i j}>0$. Hence, we proved that, if $I=\emptyset$ then there exists a capacity $\mu$ such that $i, j \in N, I_{i j}^{\mu}>0$, i.e., there exists a possible positive synergy between pair of criteria $\{i, j\}$. Hence, there is no necessary independence between criteria $i$ and $j$.

The following example illustrates the two previous results in this section.

Example 1. $N=\{1,2,3\}, P=\left\{\left(a_{23}, a_{1}\right),\left(a_{12}, a_{23}\right)\right\}$.

The ordinal preference information $\{P, I\}$ contains no indifference and the binary relation $(P \cup M)$ contains no strict cycle, so $\{P, I\}$ is representable by a 2-maxitive Sugeno integral model. A suitable topological sorting on $(P \cup M)$ is given by: $\mathcal{B}_{0}=\left\{a_{0}\right\} ; \mathcal{B}_{1}=\left\{a_{1}, a_{2}, a_{3}\right\} ; \mathcal{B}_{2}=\left\{a_{13}, a_{23}\right\} ; \mathcal{B}_{3}=\left\{a_{12}\right\}$. The preference information $\{P, I\}$ is representable by the capacity $\mu$ given by Table 3. We can see that $I_{i j}^{\mu}>0, \forall i, j \in N$.:

\begin{tabular}{|c|c|c|c|c|c|}
\hline $\bar{S}$ & $\emptyset\{1\}\{2\}\{3\}$ & $\{1,3\}$ & $\{2,3\}$ & $\{1,2\}$ & $\{1,2,3\}$ \\
\hline$\overline{\mu(S)}$ & $\begin{array}{lllll}0 & 1 / 3 & 1 / 3 & 1 / 3\end{array}$ & $2 / 3$ & $2 / 3$ & 1 & 1 \\
\hline$I_{\mu(S)}^{S}$ & -- & $2 / 3$ & $2 / 3$ & 1 & - \\
\hline
\end{tabular}


Table 3: The capacity $\mu$ and the corresponding interaction indices.

\section{A MILP testing necessary interactions}

In this section, we relaxe the hypothesis that we only ask a preference information on binary alternatives since the set of binary alternatives is restrictive. Given two criteria $i$ and $j$, we elaborate a MILP to test in two steps if a preference information on the set of alternatives is representable by a 2-maxitive Sugeno integral model. Then, in the third step, we test the existence of a necessary null or positive interaction between $i$ and $j$. In the next subsection, we show how to linearize the min and max functions so as to obtain a MILP.

\subsection{Linearization of min and max functions}

Given $n$ real numbers $x_{1}, x_{2}, \cdots, x_{n}$, we have:

- $m=\min \left(x_{1}, x_{2}, \cdots, x_{n}\right) \Longleftrightarrow m \leq x_{i} ; m \geq x_{i}-A \delta_{i} ; \delta_{i} \in\{0,1\} \forall i=$ $1,2, \cdots, n ; \delta_{1}+\delta_{2}+\cdots+\delta_{n}=n-1$ and $A$ is a "big" positive constant arbitrarily chosen.

- $M=\max \left(x_{1}, x_{2}, \cdots, x_{n}\right) \Longleftrightarrow M \geq x_{i} ; M \leq x_{i}+B \delta_{i} ; \delta_{i} \in\{0,1\}$

$\forall i=1,2, \cdots, n ; \delta_{1}+\delta_{2}+\cdots+\delta_{n}=n-1$ and $B$ is a "big" positive constant arbitrarily chosen.

This transformation of the min and max functions into linear constraints allows us to transform the following program into a MILP.

\subsection{Algorithm}

Step 1. The following MILP $\left(M I P L_{1}\right)$ models each preference of $\{P, I\}$ by introducing two nonnegative slack variables $\alpha_{x y}^{+}$and $\alpha_{x y}^{-}$in the corresponding constraints (Equations (1a) and (1b)). Equation (1c) (resp. (1d) ) ensures the normalization (resp. monotonicity) of capacity $\mu$. Equation (1e) reflects 2maxitivity condition. The objective function $Z_{1}$ minimizes all the nonnegative variables introduced in $(1 a)$ and $(1 b)$.

Minimize $Z_{1}=\sum_{(x, y) \in P \cup I}\left(\alpha_{x y}^{+}+\alpha_{x y}^{-}\right)$

Subject to

$S_{\mu}(u(x))-S_{\mu}(u(y))+\alpha_{x y}^{+}-\alpha_{x y}^{-} \geq \varepsilon \forall x, y \in X$ such that $x P y$

$S_{\mu}(u(x))-S_{\mu}(u(y))+\alpha_{x y}^{+}-\alpha_{x y}^{-}=0 \quad \forall x, y \in X$ such that $x I y$

$\alpha_{x y}^{+} \geq 0, \quad \alpha_{x y}^{-} \geq 0 \forall x, y \in X$ such that $x(P \cup I) y$

$\varepsilon \geq 0$

$\mu(N)=1$

$\mu_{i} \geq 0, \mu_{i j} \geq \mu_{i}, \mu_{i j} \geq \mu_{j}$, for all $i, j \in N$.

$\mu(S)=\bigvee_{i, j \in S} \mu_{i j} \quad \forall S \subseteq N,|S| \geq 3$ 
The MILP $\left(M I P L_{1}\right)$ is always feasible due to the introduction of the nonnegative variables $\alpha_{x y}^{+}$and $\alpha_{x y}^{-}$. There are two possible cases:

1. If the optimal solution of $\left(M I P L_{1}\right)$ is $Z_{1}^{*}=0$, then we can conclude that, depending on the sign of the variable $\varepsilon$, the preference information $\{P, I\}$ may be representable by a 2-maxitive Sugeno integral model. The next step of the procedure, Step 2 hereafter, will confirm or not this possibility.

2. If the optimal solution of $\left(M I P L_{1}\right)$ is $Z_{1}^{*}>0$, then there is no 2-maxitive Sugeno integral model compatible with $\{P, I\}$.

Step 2. Here, the MILP $\left(M I P L_{2}\right)$ ensures the existence of a 2-maxitive Sugeno integral model compatible with $\{P, I\}$, when the optimal solution of $\left(P L_{1}\right)$ is $Z_{1}^{*}=0$. Compared to the previous MILP, in this formulation, we only removed the nonnegative variables $\alpha_{x y}^{+}$and $\alpha_{x y}^{-}$(or put them equal to zero) and change the objective function by maximizing the value of the variable $\varepsilon$, in order to satisfy the strict preference relation.

Maximize $Z_{2}=\varepsilon$

Subject to

$S_{\mu}(u(x))-S_{\mu}(u(y)) \geq \varepsilon \forall x, y \in X$ such that $x P y$

$S_{\mu}(u(x))-S_{\mu}(u(y))=0 \forall x, y \in X$ such that $x I y$

$\varepsilon \geq 0$

$\mu(N)=1$

$\mu_{i} \geq 0, \mu_{i j} \geq \mu_{i}, \mu_{i j} \geq \mu_{j}$ for all $i, j \in N$.

$\mu(S)=\bigvee_{i, j \in S} \mu_{i j} \quad \forall S \subsetneq N,|S| \geq 3$

Notice that $\left(M I P L_{2}\right)$ is solved only if $Z_{1}^{*}=0$. Hence, the linear program $\left(M I P L_{2}\right)$ is always feasible and it does not have an unbounded solution (it is not restrictive to suppose that $\left.S_{\mu}(u(x)) \in[0,1] ; \forall x \in X\right)$. Hence, we have one of the following two cases:

1. If the linear program $\left(M I P L_{2}\right)$ is feasible with optimal solution $Z_{2}^{*}=0$, then there is no 2-maxitive Sugeno integral model compatible with $\{P, I\}$.

2. If the optimal solution of is $\left(M I P L_{2}\right)$ is $Z_{2}^{*}>0$, then ordinal information $\{P, I\}$ is representable by a 2 -maxitive Sugeno integral model.

Step 3. At this step, we suppose that the preference information $\{P, I\}$ is representable by a 2 -maxitive Sugeno integral model, i.e., $Z_{2}^{*}>0$. In order to know if the interaction between $i$ and $j$ is necessarily null (resp. positive) w.r.t. the provided preference information. At $\left(P L_{2}\right)$, we add the contraint $I_{i j}^{\mu}>0$ (resp. $\left.I_{i j}^{\mu}=0\right)$ and we obtain the MILP denoted by $M I P L_{N N}^{i j}\left(\operatorname{resp} . M I P L_{N P}^{i j}\right)$. After a resolution of the MILP, we have one of the following three possible conclusions : 
1. If $M I P L_{N N}^{i j}$ (resp. $M I P L_{N P}^{i j}$ ) is not feasible, then there is a necessary positive (resp. null) interaction between $i$ and $j$. Indeed, as the program $\left(M I P L_{2}\right)$ is feasible with an optimal solution, the contradiction about the representation of $\{P, I\}$ only comes from the introduction of the constraint $I_{i j}^{\mu}>0$ (resp. $I_{i j}^{\mu}=$ $0)$.

2. If $M I P L_{N N}^{i j}$ (resp. $M I P L_{N P}^{i j}$ ) is feasible and the optimal solution $Z_{3}^{*}=0$, then the contraint $S_{\mu}(u(x))-S_{\mu}(u(y)) \geq \varepsilon \forall x, y \in X$ such that $x P y$ is satisfied with $\varepsilon=0$, i.e., it is not possible to model strict preference by adding the constraint $I_{i j}^{\mu}>0\left(\right.$ resp. $\left.I_{i j}^{\mu}=0\right)$ in $M I P L_{N N}^{i j}\left(\right.$ resp. $\left.M I P L_{N P}^{i j}\right)$. Therefore, we can conclude that there is a necessary positive (resp. null) interaction between $i$ and $j$.

3. If $M I P L_{N N}^{i j}$ (resp. $M I P L_{N P}^{i j}$ ) is feasible and the optimal solution $Z_{3}^{*}>0$, then there is no necessary null (resp. positive) interaction between $i$ and $j$.

\subsection{Example}

We consider the preferences given by the DM in the classic example given by Table 1. We proved in Section 3 that these preferences are representable by a 2-maxitive Sugeno integral. The following $M I P L_{M S}^{N N}$ corresponding to the test of the existence of a necessary null interaction between the Mathematics (1) and Statistics (3):

Maximize $Z_{3}=\varepsilon$

\section{Inputs of example}

$a_{1}=0.3 ; a_{2}=0.25 ; a_{3}=0.6 ; b_{1}=0.3 ; b_{2}=0.6 ; b_{3}=0.25 ; c_{1}=0.7 ;$ $c_{2}=0.25 ; c_{3}=0.6 ; d_{1}=0.7 ; d_{2}=0.6 ; d_{3}=0.25$;

$S_{\mu}(a) \geq S_{\mu}(b)+\varepsilon ; S_{\mu}(d) \geq S_{\mu}(c)+\varepsilon ; \varepsilon \geq 0.1$

Constraints related of linearization of $S_{\mu}(x)=\max \left(\alpha_{x 1}, \alpha_{x 2}, \alpha_{x 3}, \alpha_{x 12}, \alpha_{x 13}, \alpha_{x 23}\right)$ with the introduction of binary variables $\delta_{1}^{x}, \delta_{2}^{x}, \delta_{3}^{x}, \delta_{12}^{x}, \delta_{13}^{x}, \delta_{23}^{x}$, where $x \in\{a, b, c, d\}$.

$S_{\mu}(x) \geq \alpha_{x 1} ; S_{\mu}(x) \geq \alpha_{x 2} ; S_{\mu}(x) \geq \alpha_{x 3} ; S_{\mu}(x) \geq \alpha_{x 12} ; S_{\mu}(x) \geq \alpha_{x 13} ;$

$S_{\mu}(x) \geq \alpha_{x 23} ; S_{\mu}(x) \leq \alpha_{x 1}+500 \delta_{1}^{x} ; S_{\mu}(x) \leq \alpha_{x 2}+500 \delta_{2}^{x}$;

$S_{\mu}(x) \leq \alpha_{x 3}+500 \delta_{3}^{x} ; S_{\mu}(x) \leq \alpha_{x 12}+500 \delta_{12}^{x} ; S_{\mu}(x) \leq \alpha_{x 13}+500 \delta_{13}^{x} ; S_{\mu}(x) \leq$ $\alpha_{x 23}+500 \delta_{23}^{x} ; \delta_{1}^{x}+\delta_{2}^{x}+\delta_{3}^{x}+\delta_{12}^{x}+\delta_{13}^{x}+\delta_{23}^{x}=5 ; \delta_{1}^{x}, \delta_{2}^{x}, \delta_{3}^{x}, \delta_{12}^{x}, \delta_{13}^{x}, \delta_{23}^{x} \in\{0,1\}$.

Constraints related of linearization of $\alpha_{x i}=\min \left(x_{i}, \mu_{i}\right)$ with the introduction of binary variables $\delta_{i 1}^{x}, \delta_{i 2}^{x}$, where $x \in\{a, b, c, d\}$ and $i \in$ $\{1,2,3\}$.

$\alpha_{x i} \leq x_{i} ; \alpha_{x i} \leq \mu_{i} ; \alpha_{x i} \geq x_{i}-500 \delta_{i 1}^{x} ; \alpha_{x i} \geq \mu_{i}-500 \delta_{i 2}^{x} ; \delta_{i 1}^{x}+\delta_{i 2}^{x}=1$;

$\delta_{i 1}^{x}, \delta_{i 2}^{x} \in\{0,1\}$. 
Constraints related of linearization of $\alpha_{x i j}=\min \left(x_{i}, x_{j}, \mu_{i j}\right)$ with the introduction of binary variables $\delta_{i j 1}^{x}, \delta_{i j 2}^{x}, \delta_{i j 3}^{x}$, where $x \in\{a, b, c, d\}$ and $i, j \in\{1,2,3\}, i \neq j$.

$\alpha_{x i j} \leq x_{i} ; \alpha_{x i j} \leq x_{j} ; \alpha_{x i j} \leq \mu_{i j} ; \alpha_{x i j} \geq x_{i}-500 \delta_{i j 1}^{x} ; \alpha_{x i j} \geq x_{j}-500 \delta_{i j 2}^{x} ;$

$\alpha_{x i j} \geq \mu_{i j}-500 \delta_{i j 3}^{x} ; \delta_{i j 1}^{x}+\delta_{i j 2}^{x}+\delta_{i j 3}^{x}=2 ; \delta_{i j 1}^{x}, \delta_{i j 2}^{x}, \delta_{i j 3}^{x} \in\{0,1\}$.

\section{2-maxitivity constraints}

$\mu_{12} \geq \mu_{1} ; \mu_{12} \geq \mu_{2} ; \mu_{13} \geq \mu_{1} ; \mu_{13} \geq \mu_{3} ; \mu_{23} \geq \mu_{2} ; \mu_{23} \geq \mu_{3} ; \mu_{123}=\max \left(\mu_{12}, \mu_{13}, \mu_{23}\right)$.

Contraint of normalization $\mu_{123}=1$.

Constraints related of linearization of $\mu_{123}=\max \left(\mu_{12}, \mu_{13}, \mu_{23}\right)$ with the introduction of binary variables $\delta^{1}, \delta^{2}, \delta^{3}$.

$\mu_{123} \geq \mu_{12} ; \mu_{123} \geq \mu_{13} ; \mu_{123} \geq \mu_{23} ; \mu_{123} \leq \mu_{12}+500 \delta^{1} ; \mu_{123} \leq \mu_{13}+500 \delta^{2} ; \mu_{123} \leq$ $\mu_{23}+500 \delta^{3} ; \delta^{1}+\delta^{2}+\delta^{3}=2 ; \delta^{1}, \delta^{2}, \delta^{3} \in\{0,1\}$.

Constraints related of linearization of $I_{13}^{\mu}>0$

$\mu_{13} \geq \mu_{1}+\varepsilon ; \mu_{13} \geq \mu_{3}+\varepsilon ; \varepsilon \geq 0.1$.

The results obtained by solving $M I P L_{N N}^{M S}$ are given in Table 4 . We can conclude that the interaction between Mathematics and Statistics is not necessarily null, because the optimal solution of the program $M I P L_{N N}^{M S}$ is $Z_{3}^{*}=0.1>0$. Besides, we have $S_{\mu}(a)=0.4, S_{\mu}(b)=0.3, S_{\mu}(c)=0.5$ and $S_{\mu}(d)=0.6$.

\begin{tabular}{|c|c|c|c|c|c|c|c|c|}
\hline & \multicolumn{2}{|c|}{$Z_{3}=\varepsilon 1$} & \multicolumn{2}{|c|}{23} & \multicolumn{2}{|c|}{1,2}$\{1,3\}$ & \multicolumn{2}{|c|}{$\overline{\{2,3\}\{1,2,3\}}$} \\
\hline Optimal solution $Z_{3}^{*}$ & 0.1 & - & 2 & - & - & - & - & \\
\hline Capacity $\mu$ & - & 0 & 0 & 0.4 & 0.6 & 0.5 & 1 & 1 \\
\hline Interaction index $I_{i j}^{\mu}$ & - & - & - & - & 0.6 & 0.5 & 1 & - \\
\hline
\end{tabular}

Table 4: Results of $M I P L_{N N}^{M S}$ testing necessary null interaction between Mathematics and Statistics

\section{Conclusion}

This article proposes and studies the notion of interaction between two criteria in a 2-maxitive Sugeno integral model. We make a restriction in the case where the DM gives preference information on a set of finite number of alternatives. The 2-maxitive capacity that is elicited in such a setting is not unique. The interpretation of the interaction effects between two criteria requires some caution. Indeed, we have give some examples in which the sign of the interaction index depends upon the arbitrary choice of a capacity within the set of all 2-maxitive capacities compatible with the preference information. Only necessary interactions are robust since their sign and, hence, interpretation, does not vary within the set of all representing capacities. 
Within the framework of binary alternatives, our first result gives a necessary and sufficient condition for an ordinal preference information containing no indifference to be representable by a 2-maxitive Sugeno integral model. This result is similar to that obtained in our paper on the general Choquet integral model (see Proposition 1 on [12]).

Under the conditions of our first result, in the framework of binary alternatives, if the ordinal preference information contains no indifference, our second result shows that it is always possible to represent it by a 2-maxitive Sugeno integral model which all interaction indices between two criteria are strictly positive. This result is similar to that obtained in paper on the general Choquet integral model (see Proposition 2 on [12]).

Outside the framework of binary alternatives, using the linearization of the min and max functions, we propose a MILP allowing to test whether the interpretation of the interaction indices is ambivalent or not.

The subject of this paper offer several avenues for future research.

In fact, this paper proposes an interaction index for the 2-maxitive Sugeno integral, it would be interesting to propose others for the k-maxitive Sugeno integral, with $k \geq 3$.

The notion of interaction would deserve further study. In particular, it would be interesting to have a definition that would not depend on a particular aggregation technique or on a particular index.

It would finally be interesting to study the case of bipolar scales. We are already investigating some of these research avenues.

\section{References}

1. G. Beliakov. Construction of aggregation functions from data using linear programming. Fuzzy Sets and Systems, 160:65-75, 012009.

2. G. Beliakov, Bustince S. Humberto, and Calvo S. Tomasa. A Practical Guide to Averaging Functions. 2016.

3. T. Calvo and Bernard de Baets. Aggregation operators defined by k-order additive/maxitive fuzzy measures. International Journal of Uncertainty, Fuzziness and Knowledge-Based Systems, 06(06):533-550, 1998.

4. G. Choquet. Theory of capacities. Annales de l'Institut Fourier, 5:131-295, 1954.

5. M. Couceiro, Didier Dubois, Henri Prade, and Tamas Waldhauser. Decisionmaking with Sugeno integrals. Order, 33(3):517-535, 2016.

6. Miguel Couceiro, Didier Dubois, Hélène Fargier, Michel Grabisch, Henri Prade, and Agnès Rico. New directions in ordinal evaluation: Sugeno integrals and beyond. In M. Doumpos, J.R. Figueira, S. Greco, and C. Zopounidis, editors, New Perspectives in Multiple Criteria Decision Making. Springer, Cham, April 2019.

7. D. Dubois, Jean-Luc Marichal, Henri Prade, Marc Roubens, and Régis Sabbadin. The use of the discrete Sugeno integral in decision-making: A survey. International Journal of Uncertainty, Fuzziness and Knowledge-Based Systems, 9:539-561, 10 2001.

8. M. Gondran and M. Minoux. Graphes et algorithmes. Eyrolles, Paris, 3e édition, 1995. 
9. M. Grabisch. The application of fuzzy integrals in multicriteria decision making. Fuzzy sets and systems, 1995.

10. M. Grabisch. A new algorithm for identifying fuzzy measures and its application to pattern recognition. In Proceedings of 1995 IEEE International Conference on Fuzzy Systems., volume 1, pages 145-150 vol.1, 1995.

11. M. Grabisch, Jean-Luc Marichal, Radko Mesiar, and Endre Pap. Aggregation Functions (Encyclopedia of Mathematics and Its Applications). Cambridge University Press, USA, 1st edition, 2009.

12. P.A. Kaldjob Kaldjob, Brice Mayag, and Denis Bouyssou. Necessary and Possible Interaction Between Criteria in a General Choquet Integral Model. In Information Processing and Management of Uncertainty in Knowledge-Based Systems, pages 457-466. June 2020.

13. J.L. Marichal. An axiomatic approach of the discrete Sugeno integral as a tool to aggregate interacting criteria in a qualitative framework. IEEE Transactions on Fuzzy Systems, 9(1):164-172, 2001.

14. B. Mayag and Denis Bouyssou. Necessary and possible interaction between criteria in a 2-additive Choquet integral model. European Journal of Operational Research, 283:308-320, 2019.

15. B. Mayag, Michel Grabisch, and Christophe Labreuche. A representation of preferences by the Choquet integral with respect to a 2-additive capacity. Theory and Decision, 71(3):297-324, Sep 2011.

16. J.P. Pignon and C. Labreuche. A methodological approach for operational and technical experimentation based evaluation of systems of systems architectures. In Int. Conference on Software $\&$ Systems Engineering and their Applications (ICSSEA), Paris, France, December 4-6 2007.

17. M. Radko. Generalizations of k-order additive discrete fuzzy measures. Fuzzy Sets and Systems, 102(3):423-428, 1999. Fuzzy Measures and Integrals.

18. M. Sugeno. Theory of fuzzy integrals and its applications. PhD thesis, Tokyo Institute of Technology, 1974. 